Old Dominion University

ODU Digital Commons

Electrical \& Computer Engineering Faculty

Publications

Electrical \& Computer Engineering

2005

Performance Analysis and Validation of a Recoverable Flight Control System in a Simulated Neutron Environment

Hong Zhang

W. Steven Gray

Oscar R. Gonzalez

Follow this and additional works at: https://digitalcommons.odu.edu/ece_fac_pubs

Part of the Computer Sciences Commons, Controls and Control Theory Commons, Navigation,

Guidance, Control and Dynamics Commons, and the Systems Engineering and Multidisciplinary Design

Optimization Commons 


\title{
Performance Analysis and Validation of a Recoverable Flight Control System in a Simulated Neutron Environment
}

\author{
Hong Zhang*, W. Steven Gray ${ }^{\dagger}$ and Oscar R. González ${ }^{\ddagger}$ \\ Old Dominion University, Norfolk, VA 23529-0246, U.S.A.
}

\begin{abstract}
This paper introduces a class of stochastic hybrid models for the analysis of closedloop control systems implemented with NASA's Recoverable Computer System. Such Recoverable Computer Systems have been proposed to insure reliable control performance in harsh environments. The stochastic hybrid models consist of either a stochastic finitestate automaton or a finite-state machine driven by a Markov input, which in turn drives a switched linear discrete-time dynamical system. Their stability and output tracking performance are analyzed using an extension of the existing theory for Markov jump-linear systems. For illustration, a stochastic hybrid model is used to calculate the tracking error performance of a Boeing 737 at cruising altitude and in closed-loop with a Recoverable Computer System subject to neutron-induced single-event upsets. The upsets are modeled with a Markov process. The results are validated using experimental data obtained from a simulated neutron environment in NASA's SAFETI Laboratory.
\end{abstract}

\section{Introduction}

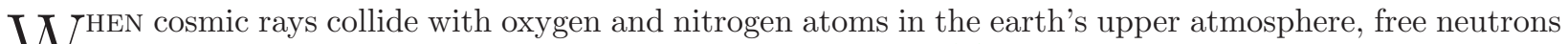
$W$ are produced with energies varying from $10 \mathrm{MeV}$ to $1 \mathrm{GeV} .^{1,2}$ The higher the altitude, the higher the neutron flux and energy. ${ }^{3}$ When a neutron passes through a solid state device, it has been observed that stored electric charge can be locally redistributed, which may cause a single-event effect (SEE). If this charge resides in a solid state memory device, for example a computer's cache memory, a binary "one" can be flipped to a "zero" or vice versa. It is also possible for dielectric material in the device to rupture and create short circuits, which can burn out neighboring devices if sufficient electric potential exists. ${ }^{4,5}$ Normally, the errors due to SEE's are classified into two categories: soft errors and hard errors. ${ }^{6,7}$ Soft errors by their nature are nondestructive to the hardware but alter memory contents and/or computer logic. Their effects are usually transient, but may require the hardware to be reset or refreshed. Hard errors, on the other hand, may be destructive. Chip burnouts cause permanent malfunctioning of the hardware. Another hard error is latchup, which is a potentially destructive short-circuit that can be corrected by resetting the hardware. Single event upsets (SEU's) are defined as soft errors, induced by radiation, that produce a malfunction at some level in the system, which is usually transient and nondestructive to the hardware. However, if a sequence of bit errors is not detected and corrected, it can cause system errors and reduce closed-loop performance. The phenomenon has been studied extensively at the component level by semiconductor manufacturers. ${ }^{4,8,9}$ In this context, both analytical models and data are available to provide SEU probability estimates under a wide variety of circumstances. In contrast, at the system level, the problem has not received much attention. In Refs. 10,11, the effect of upsets on system stability was studied for a few fault-tolerant architectures. However, at present no performance analysis studies are available.

As more commercial aircraft control systems and avionics are implemented using embedded digital hardware, SEU's have recently come to the attention of the Federal Aviation Administration (FAA) as a potential

\footnotetext{
*Ph.D. Candidate, Systems Research Laboratory, Department of Electrical and Computer Engineering, hzhang@odu.edu

${ }^{\dagger}$ The corresponding author. Associate Professor, Systems Research Laboratory, Department of Electrical and Computer Engineering, phone/fax: 757-683-4671/3220, gray@ece.odu.edu

${ }_{\ddagger}$ Associate Professor, Systems Research Laboratory, Department of Electrical and Computer Engineering, gonzalez@ece.odu.edu
} 
safety hazard. In response, a program has been developed with the general goal of quantifying the nature of the risk and to produce guidelines for the aerospace industry and chip manufacturers. As part of this program, the authors in cooperation with the NASA Langley Research Center are investigating the effects of atmospheric neutrons on commercial and experimental flight control hardware in closed-loop with aircraft simulation software. A set of experiments is underway at the Los Alamos Neutron Science Center (LANSCE) in Los Alamos, New Mexico to provide data. While a variety of different technologies will be tested, the focus here is on an experimental Recoverable Computer System (RCS) developed by Honeywell, Inc. The error recovery system in this prototype is implemented using dual-lock-step processors together with new fault tolerant architectures and communication subsystems. ${ }^{12,13}$ It has most recently been used to study recovery from transient faults introduced by high intensity electromagnetic radiation. ${ }^{14,15}$ The error recovery technique implemented on the RCS is a variation of rollback recovery; ${ }^{16,17}$ it has the following steps: checkpointing, faulttolerant comparison, rollback, and retry. During a checkpoint, the state of each microprocessor module is stored. When an upset is detected, rollback of both microprocessor modules to a previous checkpoint takes place, and then the system is allowed to proceed with normal execution. But once the execution of the normal control program is interrupted, the execution of a different control law takes place, one that has significantly different dynamics and is on a time scale that can alter the overall closed-loop dynamics of the flight control system. These characteristics of the recoverable system can be modeled as a two-mode switched system. If there is no upset detected, the system is operating in its nominal mode. When the controller is executing a rollback recovery, the system is performing in

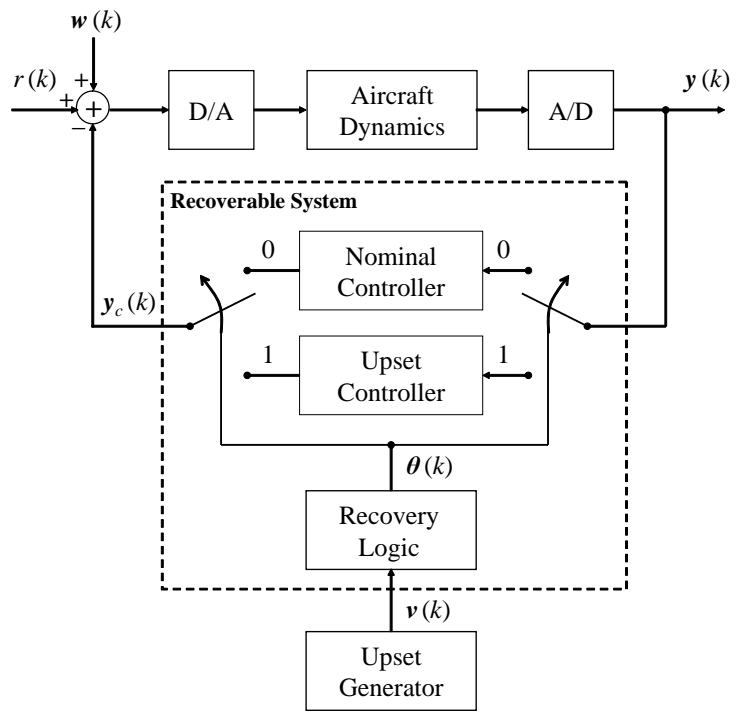

Figure 1. A conceptual closed-loop flight control system with a recoverable flight control computer. its recovery mode. A conceptual diagram of the RCS in a closed-loop configuration is shown in Fig. 1.

The FAA/NASA research program is centered around the analysis of experimental data collected from a series of experiments conducted at LANSCE. A conceptual diagram of the testbed is shown in Fig. 2. A beam of free neutrons is directed through a flux sensor at the device under test, in this case a flight control computer. The energy spectrum of the neutron source is shown in Fig. 3. Its shape is very similar to that produced by atmospheric neutrons, but the flux is five to six orders of magnitude higher. The flight control computer in this setup runs a control program which processes outputs from a Boeing 737 flight simulation system running on a separate host computer. The flight control computer generates the appropriate control signals to the aircraft simulation model for maintaining straight and level flight at a cruising altitude of 34,000 feet. This interconnection between the flight control computer and the flight simulation host computer constitutes a closedloop feedback control system, which is the unique feature of these experiments. Under nominal conditions, i.e., no neutrons, this system regulates the aircraft heading and orientation very well, even under considerable winds and gusts which can be introduced into the sim-

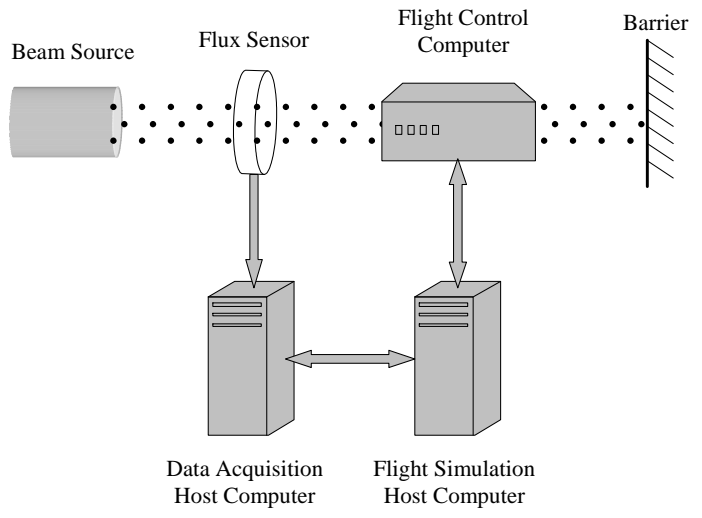

Figure 2. The testbed for the LANSCE experiments. ulation model. The data acquisition system is maintained on a third computer system. It collects the flight data from the simulation as well as the measurements from the flux sensor for off-line analysis. Should the aircraft deviate from the nominal flight path at any time, it will be possible to determine the total radiation dose the flight control computer received up to that instant. When neutrons 
collide with the flight control computer, the specific effects of any disturbance will depend on the particular nature of the control computer's internal hardware and any fault-tolerant features it possesses.

The main goal of this paper is to model and predict the output tracking error produced by neutron induced SEU's. To this end, a class of stochastic hybrid models is introduced to describe recoverable flight control systems. Both stochastic finite-state automata (SFSA's) and finite-state machines (FSM's) are used to model the recovery process of the RCS. In each case, the mean-square stability and output tracking performance of the hybrid model class are analyzed. The model is validated in a controlled experiment at NASA's SAFETI Laboratory, where computer upsets are injected into the RCS at a specified rate, and the effects on the output tracking performance of the simulated aircraft are directly observed and quantified. The output performance is compared with the theoretical predictions using the SFSA and FSM models. A separate publication will address the model's performance against the LANSCE data. Since the LANSCE neutron source is much more intense than normal atmospheric sources,

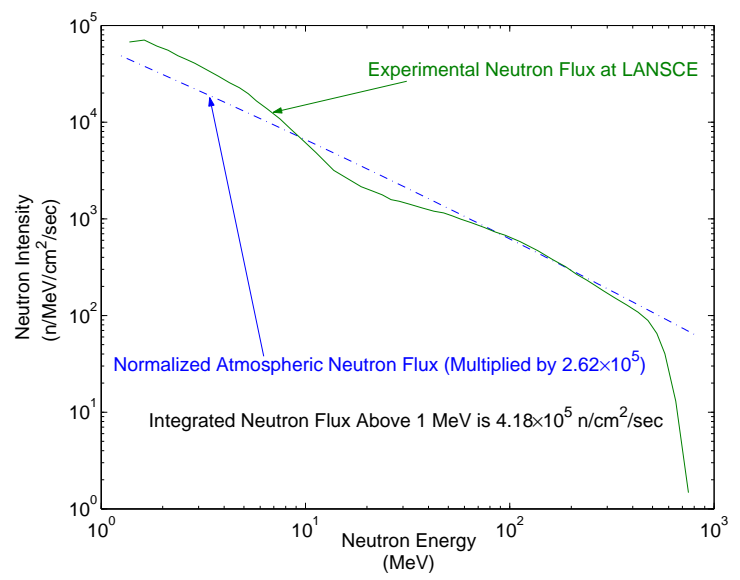

Figure 3. The neutron energy spectrum at LANSCE in December, 2002. the final application of these models will be to rescale the performance predictions made using the LANSCE data to fit aircraft flying in normal operating conditions.

This paper is organized as follows. Sections II and III provide the main theoretical background. Section II describes the general class of stochastic hybrid models. Section III provides the mathematical tools for analyzing the stability and output performance of these hybrid models. Sections IV and V employ these tools to analyze a recoverable flight control system. Specifically, Section IV describes how to model a Boeing 737 system in closed-loop with an RCS. Section V provides the stability and output performance predictions of the flight control system when subject to SEU's. Section VI describes the validation of the model using data obtained from the simulated-neutron environment at NASA Langley. Section VII summarizes the conclusions and plans for future research.

The mathematical notation used throughout this paper is largely consistent with Ref. 18. Random variables are denoted in bold italic fonts. The symbol $\mathbb{Z}^{+}$denotes the set of all non-negative integers. $\mathbb{N}$ is the set of all natural numbers. $\mathbb{R}^{n}$ is the $n$-dimensional real vector space, and $\mathbb{M}\left(\mathbb{R}^{n}\right)$ is the normed linear space of all $n \times n$ real matrices. The subset of all symmetric positive semi-definite matrices is $\mathbb{M}\left(\mathbb{R}^{n}\right)^{+}$. $\mathbb{H}_{N}^{n}=\left\{V=\left(V_{1}, V_{2}, \ldots, V_{N}\right): V_{i} \in \mathbb{M}\left(\mathbb{R}^{n}\right)\right\}$ will be used to denote the space of all $N$-tuples of $n \times n$ real matrices. If every $V_{i}$ of a given $V$ in $\mathbb{H}_{N}^{n}$ is positive definite or positive semi-definite, this is indicated, respectively, by $V>0$ and $V \geq 0 . \mathbb{H}_{N}^{n+}$ denotes the set $\left\{V \in \mathbb{H}_{N}^{n}: V \geq 0\right\}$. Given $U, V \in \mathbb{H}_{N}^{n}$, the inner product on $\mathbb{H}_{N}^{n}$ is defined by

$$
\langle U, V\rangle=\sum_{i=1}^{N} \operatorname{tr}\left(U_{i}^{\mathrm{T}} V_{i}\right),
$$

and $\|V\|^{2}=\langle V, V\rangle$ is the induced norm squared of $V$. $(\|\cdot\|$ will also be used for representing the standard norm on $\mathbb{R}^{n}$.) $\mathbb{B}\left(\mathbb{H}_{N}^{n}\right)$ is the space of all bounded linear operators on $\mathbb{H}_{N}^{n}$ under the induced operator norm

$$
\|\mathcal{L}\|=\sup _{V \neq 0} \frac{\|\mathcal{L}(V)\|}{\|V\|}
$$

where $\mathcal{L} \in \mathbb{B}\left(\mathbb{H}_{N}^{n}\right) . r_{\sigma}(\mathcal{L})$ is used to denote the spectral radius of $\mathcal{L}$, specifically, $r_{\sigma}(\mathcal{L})=\lim _{k \rightarrow \infty}\left\|\mathcal{L}^{k}\right\|^{\frac{1}{k}}$ for $k \in \mathbb{Z}^{+}$.

\section{Stochastic Hybrid Models for Recoverable Closed-Loop Systems}

A class of stochastic hybrid models for an aircraft in closed-loop with an RCS is shown in Fig. 4. It A consists of a stochastic hybrid system driven by an SFSA with a Markov input process. Similar to electromagnetic radiation induced upsets (see Ref. 10), the neutron induced upset process can be modeled 
as a two-state Markov chain. The SFSA models the rollback recovery process. The switched system models the dynamics of the closed-loop due to the presence of controller recoveries. If the switching process $\boldsymbol{\theta}(k)$ is a Markov chain, then the system's mean-square stability and output performance can be analyzed using existing methods (for example, see Refs. 19,20). However, in most cases, the output process of an SFSA is not Markovian. The goal of this section is to describe a related process which is Markov.

In the present context, an SFSA is defined as follows. $^{21,22,23}$

Definition 1 (SFSA). Let $(\Omega, \mathcal{F}, \operatorname{Pr})$ represent the ambient probability space. A stochastic finite-state automaton $\mathcal{A}$ is a six-tuple, $\left(\Sigma_{I}, \Sigma_{S}, \Sigma_{O},[0,1], f, g\right)$, where $\Sigma_{I}=\left\{\eta_{1}, \eta_{2}, \ldots, \eta_{M}\right\}$ is the set of input symbols; $\Sigma_{S}=$ $\left\{\zeta_{1}, \zeta_{2}, \ldots, \zeta_{N}\right\}$ denotes the set of internal states; and

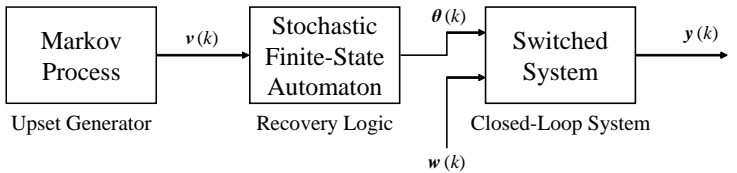

Figure 4. The stochastic hybrid model consisting of a switched system driven by an SFSA. $\Sigma_{O}=\left\{\xi_{1}, \xi_{2}, \ldots, \xi_{P}\right\}$ is the set of output symbols. Let $\boldsymbol{\nu}(k), \boldsymbol{z}(k)$, and $\boldsymbol{\theta}(k)$ denote the input random process, the state process, and the output process of the automaton, respectively, where $k \in \mathbb{Z}^{+} . f$ describes the dynamics of the state transition relation:

$$
\begin{aligned}
& f:\left(\Sigma_{S}, \Sigma_{I}, \Sigma_{S}\right) \rightarrow[0,1] \\
& \quad\left(\zeta_{j}, \eta_{l}, \zeta_{i}\right) \mapsto \operatorname{Pr}\left\{\boldsymbol{z}(k+1)=\zeta_{j} \mid\left(\boldsymbol{\nu}(k)=\eta_{l}, \boldsymbol{z}(k)=\zeta_{i}\right)\right\}:=\left[\Pi^{\eta_{l}}\right]_{\zeta_{i} \zeta_{j}},
\end{aligned}
$$

which is the $\zeta_{i} \zeta_{j}$-th component of the state transition matrix $\Pi^{\eta_{l}}$ for the input symbol $\eta_{l}$. For fixed $i \in$ $\{1,2, \ldots, N\}$ and $l \in\{1,2, \ldots, M\}, \sum_{j=1}^{N}\left[\Pi^{\eta_{l}}\right]_{\zeta_{i} \zeta_{j}}=1$. The output relation $g$ is described by

$$
\begin{aligned}
& g:\left(\Sigma_{O}, \Sigma_{I}, \Sigma_{S}\right) \rightarrow[0,1] \\
& \quad\left(\xi_{t}, \eta_{r}, \zeta_{s}\right) \mapsto \operatorname{Pr}\left\{\boldsymbol{\theta}(k)=\xi_{t} \mid\left(\boldsymbol{\nu}(k)=\eta_{r}, \boldsymbol{z}(k)=\zeta_{s}\right)\right\}:=\left[\Phi^{\eta_{r}}\right]_{\zeta_{s} \xi_{t}},
\end{aligned}
$$

where for given $s \in\{1,2, \ldots, N\}$ and $r \in\{1,2, \ldots, M\}, 0 \leq \sum_{t=1}^{P}\left[\Phi^{\eta_{r}}\right]_{\zeta_{s} \xi_{t}} \leq 1$.

Definition 2 (FSM). For an SFSA, if both $\left[\Pi^{\eta_{l}}\right]_{\zeta_{i} \zeta_{j}}$ and $\left[\Phi^{\eta_{r}}\right]_{\zeta_{s} \xi_{t}}$ only assume values 0 or 1 for all $\eta_{l}, \eta_{r} \in$ $\Sigma_{I}, \zeta_{i}, \zeta_{j}, \zeta_{s} \in \Sigma_{S}$ and $\xi_{t} \in \Sigma_{O}$, then the automaton is called a finite-state machine, and is denoted by the six-tuple $\mathcal{M}=\left(\Sigma_{I}, \Sigma_{S}, \Sigma_{O},\{0,1\}, f, g\right)$.

Therefore, an FSM is simply a special case of an SFSA. In the current application, it is always assumed that the output is isomorphic to the state of an SFSA with probability one, that is, $P \equiv N$ and $g\left(\xi_{t}, \eta_{r}, \zeta_{s}\right)=$ $\mathbf{1}_{\{s=t\}}$ for all input symbols $\eta_{r} \in \Sigma_{I}$, where $\mathbf{1}_{\{s=t\}}$ is the Dirac measure. The following theorem describes an input-output cross chain process generated by an SFSA with a Markov input. The special case for FSM's appears in Refs. 24, 25 and the references therein. A similar idea (without proof) also appears in Ref. 26 for random dynamical systems with jumps. A more general Feller-Markov property is proved in Ref. 27 for continuous-time stochastic systems.

Theorem 1. If the input process $\boldsymbol{\nu}(k)$ of an $N$-state SFSA $\mathcal{A}$ is an $M$-state Markov chain with probability transition matrix $\Pi_{I}$, which is independent of the initial state of the automaton, $\boldsymbol{z}(0)$, then the Cartesian product of the input and output processes of the SFSA, $\boldsymbol{\rho}(k):=(\boldsymbol{\nu}(k), \boldsymbol{\theta}(k))$, is an MN-state Markov chain with state transition matrix

$$
\Pi_{I / O}=\operatorname{diag}\left(\Pi^{\eta_{1}}, \Pi^{\eta_{2}}, \ldots, \Pi^{\eta_{M}}\right) \cdot\left(\Pi_{I} \otimes I_{N}\right) .
$$

(Here $I_{N}$ denotes an $N \times N$ identity matrix.)

Proof. Only an outline is given here. The complete proof is available in Ref. 28. For any $k \in \mathbb{Z}^{+}$, let for example $\{\nu(k)\}$ represent the event $\{\boldsymbol{\nu}(k)=\nu(k)\}$ and $\{(\nu(k), z(k))\}$ represent the event $\{(\boldsymbol{\nu}(k), \boldsymbol{z}(k))=(\nu(k), z(k))\}$. Consider an arbitrary event of the form

$$
\mathscr{F}(k):=\{(\nu(k), z(k)),(\nu(k-1), z(k-1)), \ldots,(\nu(0), z(0))\} .
$$


When $k \geq 2$, from the assumptions that $\boldsymbol{\nu}$ is Markov and independent of $\boldsymbol{z}(0)$, it follows that

$$
\begin{aligned}
\operatorname{Pr}\{\mathscr{F}(k)\} & =\prod_{i=0}^{k-1}\left[\Pi^{\nu(i)}\right]_{z(i) z(i+1)} \operatorname{Pr}\{\nu(k), \nu(k-1), \ldots, \nu(0), z(0)\} \\
& =\prod_{i=0}^{k-1}\left[\Pi^{\nu(i)}\right]_{z(i) z(i+1)} \operatorname{Pr}\{\nu(k) \mid \nu(k-1), \ldots, \nu(0)\} \operatorname{Pr}\{\nu(k-1), \ldots, \nu(0), z(0)\} \\
& =\left[\Pi^{\nu(k-1)}\right]_{z(k-1) z(k)} \prod_{i=0}^{k-2}\left[\Pi^{\nu(i)}\right]_{z(i) z(i+1)} \operatorname{Pr}\{\nu(k) \mid \nu(k-1)\} \operatorname{Pr}\{\nu(k-1), \ldots, \nu(0), z(0)\} \\
& =\left[\Pi^{\nu(k-1)}\right]_{z(k-1) z(k)}\left[\Pi_{I}\right]_{\nu(k-1) \nu(k)} \prod_{i=0}^{k-2}\left[\Pi^{\nu(i)}\right]_{z(i) z(i+1)} \operatorname{Pr}\{\nu(k-1), \ldots, \nu(0), z(0)\} \\
& =\left[\Pi^{\nu(k-1)}\right]_{z(k-1) z(k)}\left[\Pi_{I}\right]_{\nu(k-1) \nu(k)} \operatorname{Pr}\{\mathscr{F}(k-1)\} .
\end{aligned}
$$

Therefore,

$$
\operatorname{Pr}\{(\nu(k), z(k)) \mid \mathscr{F}(k-1)\}=\left[\Pi^{\nu(k-1)}\right]_{z(k-1) z(k)}\left[\Pi_{I}\right]_{\nu(k-1) \nu(k)} .
$$

Applying Lemma 16 in Ref. 28 (with $r=1$ ) gives

$$
\operatorname{Pr}\{\nu(k) \mid \nu(k-1), z(k-1)\}=\operatorname{Pr}\{\nu(k) \mid \nu(k-1)\}, \quad k \geq 1 .
$$

Thus,

$$
\begin{aligned}
& \operatorname{Pr}\{(\nu(k), z(k)),(\nu(k-1), z(k-1))\} \\
& =\left[\Pi^{\nu(k-1)}\right]_{z(k-1) z(k)} \operatorname{Pr}\{\nu(k), \nu(k-1), z(k-1)\} \\
& =\left[\Pi^{\nu(k-1)}\right]_{z(k-1) z(k)} \operatorname{Pr}\{\nu(k) \mid \nu(k-1), z(k-1)\} \operatorname{Pr}\{\nu(k-1), z(k-1)\} \\
& =\left[\Pi^{\nu(k-1)}\right]_{z(k-1) z(k)} \operatorname{Pr}\{\nu(k) \mid \nu(k-1)\} \operatorname{Pr}\{\nu(k-1), z(k-1)\} \\
& =\left[\Pi^{\nu(k-1)}\right]_{z(k-1) z(k)}\left[\Pi_{I}\right]_{\nu(k-1) \nu(k)} \operatorname{Pr}\{(\nu(k-1), z(k-1))\} .
\end{aligned}
$$

Consequently,

$$
\operatorname{Pr}\{(\nu(k), z(k)) \mid(\nu(k-1), z(k-1))\}=\left[\Pi^{\nu(k-1)}\right]_{z(k-1) z(k)}\left[\Pi_{I}\right]_{\nu(k-1) \nu(k)} .
$$

Comparing Eqs. (2) and (3), it is clear that

$$
\operatorname{Pr}\{(\nu(k), z(k)) \mid \mathscr{F}(k-1)\}=\operatorname{Pr}\{(\nu(k), z(k)) \mid(\nu(k-1), z(k-1))\},
$$

which implies that the input-state cross process $(\boldsymbol{\nu}, \boldsymbol{z})$ is Markov. From the assumption that the output process is isomorphic to the state process of the SFSA with probability one, it is immediate that $\boldsymbol{\rho}=(\boldsymbol{\nu}, \boldsymbol{\theta})$ is also Markov with the transition probability

$$
\operatorname{Pr}\left\{\left(\boldsymbol{\nu}(k)=\eta_{t}, \boldsymbol{\theta}(k)=\xi_{j}\right) \mid\left(\boldsymbol{\nu}(k-1)=\eta_{s}, \boldsymbol{\theta}(k-1)=\xi_{i}\right)\right\}=\left[\Pi_{I}\right]_{\eta_{s} \eta_{t}}\left[\Pi^{\eta_{s}}\right]_{\xi_{i} \xi_{j}} .
$$

The matrix of transition probabilities $\left[\Pi_{I}\right]_{\eta_{s} \eta_{t}}\left[\Pi^{\eta_{s}}\right]_{\xi_{i} \xi_{j}}$ for $i, j=1,2, \ldots, N$ and $s, t=1,2, \ldots, M$ is

$$
\Pi_{I / O}=\left[\begin{array}{ccc}
{\left[\Pi_{I}\right]_{\eta_{1} \eta_{1}} \Pi^{\eta_{1}}} & \ldots & {\left[\Pi_{I}\right]_{\eta_{1} \eta_{M}} \Pi^{\eta_{1}}} \\
\vdots & & \vdots \\
{\left[\Pi_{I}\right]_{\eta_{M} \eta_{1}} \Pi^{\eta_{M}}} & \cdots & {\left[\Pi_{I}\right]_{\eta_{M} \eta_{M}} \Pi^{\eta_{M}}}
\end{array}\right]=\operatorname{diag}\left(\Pi^{\eta_{1}}, \Pi^{\eta_{2}}, \ldots, \Pi^{\eta_{M}}\right) \cdot\left(\Pi_{I} \otimes I_{N}\right),
$$

which concludes the proof of the theorem. 


\section{Stability and Performance Analysis of Stochastic Hybrid Systems}

CONSIDER the $n$ th-order stochastic hybrid system in Fig. 4 with $p$ output signals given by the state space model

$$
\begin{aligned}
\boldsymbol{x}(k+1) & =A_{\boldsymbol{\theta}(k)} \boldsymbol{x}(k)+G_{\boldsymbol{\theta}(k)} \boldsymbol{w}(k), \quad \boldsymbol{x}(0)=\boldsymbol{x}_{0}, \boldsymbol{\theta}(0)=\boldsymbol{\theta}_{0} \\
\boldsymbol{y}(k) & =C_{\boldsymbol{\theta}(k)} \boldsymbol{x}(k),
\end{aligned}
$$

where $\boldsymbol{\theta}(k)$ is a stochastic switching signal produced by the SFSA. Here $\boldsymbol{w}(k)$ is a zero-mean, white noise process with covariance matrix $I_{m}$. In general $\boldsymbol{\theta}(k)$ is not necessarily Markov, so letting $\boldsymbol{\rho}(k)$ be the Cartesian product of the input and output processes of the SFSA (see Fig. 5), the analysis can be performed using the $n$ th-order Markov jump-linear system

$$
\begin{aligned}
\boldsymbol{x}(k+1) & =\tilde{A}_{\boldsymbol{\rho}(k)} \boldsymbol{x}(k)+\tilde{G}_{\boldsymbol{\rho}(k)} \boldsymbol{w}(k), \quad \boldsymbol{x}(0)=\boldsymbol{x}_{0}, \boldsymbol{\rho}(0)=\boldsymbol{\rho}_{0} \\
\boldsymbol{y}(k) & =\tilde{C}_{\boldsymbol{\rho}(k)} \boldsymbol{x}(k),
\end{aligned}
$$

which is equivalent to system (4) in the following sense.

Definition 3 (Model Equivalence). The stochastic hybrid system (4) and the Markov jump-linear system (5) are said to be model-equivalent if $\tilde{A}_{\boldsymbol{\rho}(k)}:=\tilde{A}_{(\boldsymbol{\nu}(k), \boldsymbol{\theta}(k))} \equiv A_{\boldsymbol{\theta}(k)}, \tilde{G}_{\boldsymbol{\rho}(k)}:=\tilde{G}_{(\boldsymbol{\nu}(k), \boldsymbol{\theta}(k))} \equiv G_{\boldsymbol{\theta}(k)}$ and $\tilde{C}_{\boldsymbol{\rho}(k)}:=$ $\tilde{C}_{(\boldsymbol{\nu}(k), \boldsymbol{\theta}(k))} \equiv C_{\boldsymbol{\theta}(k)}$ for $k \in \mathbb{Z}^{+}$.

From the definition, the following result is immediate.

Lemma 1. When systems (4) and (5) are model-equivalent, they have the same state process $\boldsymbol{x}(k)$ and the same output process $\boldsymbol{y}(k)$ for $k \in \mathbb{Z}^{+}$.

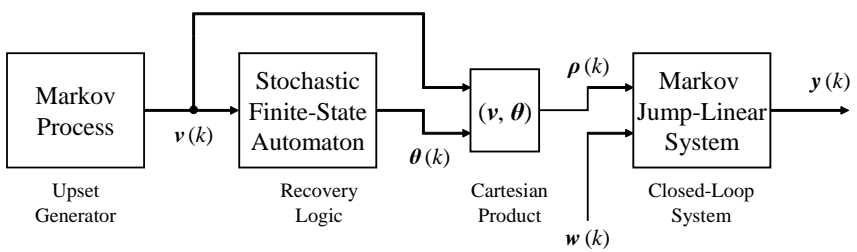

This concept is employed in the following subsection to perform stability and performance analysis of system (4).

Figure 5. The equivalent Markov jump-linear model for a stochastic hybrid system driven by an SFSA.

\section{A. Mean-Square Stability of a Stochastic Hybrid System}

The stability definition for a stochastic hybrid system used throughout is given below.

Definition 4 (Mean-Square Stability). A stochastic hybrid system (4) with $\boldsymbol{w}(k)=0$ is mean-square stable $(M S S)$ if $E\left\{\|\boldsymbol{x}(k)\|^{2}\right\} \rightarrow 0$ as $k \rightarrow \infty$ for any initial condition $\boldsymbol{x}_{0}$ with a finite second-order moment and any initial distributions for $\boldsymbol{\nu}_{0}$ and $\boldsymbol{\theta}_{0}$.

Mean-square stability of the equivalent system (5) can be determined using standard stability results for Markov jump-linear systems (see Ref. 18). For $i=1,2, \ldots, M$ and $j=1,2, \ldots, N$, let $l=(i-$ 1) $N+j$ and $\mu_{l}:=\left(\eta_{i}, \xi_{j}\right)$. The symbol set $\left\{\mu_{l}: l=1,2, \ldots, M N\right\}$ labels the elements in $\Sigma_{I} \times \Sigma_{O}$. Let $\bar{C}=\left(\bar{C}_{\mu_{1}}, \bar{C}_{\mu_{2}}, \ldots, \bar{C}_{\mu_{M N}}\right) \in \mathbb{H}_{M N}^{n+}$ be an $M N$-tuple with $\bar{C}_{\mu_{l}}=\tilde{C}_{\mu_{l}}^{\mathrm{T}} \tilde{C}_{\mu_{l}}$. The linear operators $\mathcal{E}(\cdot)=$

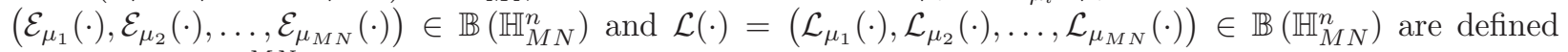
as $\mathcal{E}_{\mu_{l}}(\bar{C})=\sum_{q=1}^{M N}\left[\Pi_{I / O}\right]_{\mu_{l} \mu_{q}} \bar{C}_{\mu_{q}}$ and $\mathcal{L}_{\mu_{l}}(\bar{C})=A_{\mu_{l}}^{\mathrm{T}} \mathcal{E}_{\mu_{l}}(\bar{C}) A_{\mu_{l}}$, respectively. The operator $\mathcal{L}$ has the matrix representation

$$
\mathscr{A}_{2}:=\operatorname{diag}\left(\tilde{A}_{\mu_{1}}^{\mathrm{T}} \otimes \tilde{A}_{\mu_{1}}^{\mathrm{T}}, \tilde{A}_{\mu_{2}}^{\mathrm{T}} \otimes \tilde{A}_{\mu_{2}}^{\mathrm{T}}, \ldots, \tilde{A}_{\mu_{M N}}^{\mathrm{T}} \otimes \tilde{A}_{\mu_{M N}}^{\mathrm{T}}\right)\left(\Pi_{I / O} \otimes I_{n^{2}}\right)
$$

(see Fig. 6). From this representation and the results in Ref. 18, the following stability theorem is evident.

Theorem 2. The following statements regarding system (5) are equivalent:

(a) The Markov jump-linear system is MSS;

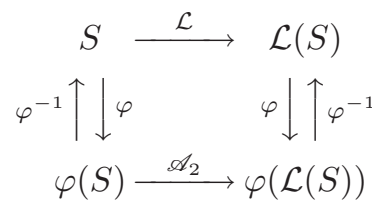

Figure 6. The relationship between $\mathcal{L}$ and $\mathscr{A}_{2}$. Here $S \in$ $\mathbb{H}_{M N}^{n+}$ and $\varphi$ denotes the column stacking operator. 
(b) $r_{\sigma}(\mathcal{L})<1$;

(c) $r_{\sigma}\left(\mathscr{A}_{2}\right)<1$.

From Lemma 1, the following result provides a stability test for the stochastic hybrid system (4).

Lemma 2. A stochastic hybrid system (4) is MSS if and only if the model-equivalent Markov jump-linear system (5) is MSS.

\section{B. Output Performance of a Stochastic Hybrid Model}

For an MSS Markov jump-linear system (5), the output performance measure $J$ is defined below:

$$
J=\left\{\begin{array}{lll}
J_{0}:=E\left\{\sum_{k=0}^{\infty}\|\boldsymbol{y}(k)\|^{2}\right\} & : & \boldsymbol{w}(k) \equiv 0 \\
J_{w}:=\lim _{k \rightarrow \infty} E\left\{\|\boldsymbol{y}(k)\|^{2}\right\} & : & \boldsymbol{w}(k) \not \equiv 0,
\end{array}\right.
$$

where $J_{0}$ is the mean output energy, and $J_{w}$ is the mean output power. The goal of this section is to produce explicit analytical expressions for $J$ via a generalization of the observability Gramian described in Refs. 18,29. This expression for $J_{w}$ can be shown to be similar to the output's power semi-norm developed in Ref. 30 for network controlled systems with data dropouts when both $\tilde{G}$ and $\tilde{C}$ in system (5) are not switched.

Suppose that $\boldsymbol{\rho}(k)$ has a stationary distribution, and $\boldsymbol{\rho}_{s}$ is a random variable with this distribution. Then $\pi_{I / O}\left(\mu_{l}\right):=\operatorname{Pr}\left\{\boldsymbol{\rho}_{s}=\mu_{l}\right\}$ for $l=1,2, \ldots, M N$ is determined by solving the eigen-equation

$$
\left[\begin{array}{c}
\pi_{I / O}\left(\mu_{1}\right) \\
\pi_{I / O}\left(\mu_{2}\right) \\
\vdots \\
\pi_{I / O}\left(\mu_{M N}\right)
\end{array}\right]=\Pi_{I / O}^{\mathrm{T}}\left[\begin{array}{c}
\pi_{I / O}\left(\mu_{1}\right) \\
\pi_{I / O}\left(\mu_{2}\right) \\
\vdots \\
\pi_{I / O}\left(\mu_{M N}\right)
\end{array}\right] .
$$

The following theorem is a variation of Proposition 8 in Ref. 19.

Theorem 3. For an MSS Markov jump-linear system (5), where $\boldsymbol{\rho}(k)$ is aperiodic and ergodic, let $\bar{Q}_{\mu_{i}}(k):=$ $E\left\{\boldsymbol{x}(k) \boldsymbol{x}^{\mathrm{T}}(k) \mathbf{1}_{\left\{\boldsymbol{\rho}(k)=\mu_{i}\right\}}\right\}$ for any $k \in \mathbb{Z}^{+}$and $\bar{Q}_{\mu_{i}}:=\lim _{k \rightarrow \infty} \bar{Q}_{\mu_{i}}(k)$. If $\boldsymbol{x}_{0}, \boldsymbol{w}$, and $\boldsymbol{\rho}$ are independent then

$$
\bar{Q}_{\mu_{i}}=\varphi_{\mu_{i}}^{-1}\left(\left(I_{M N n^{2}}-\mathscr{A}_{2}^{\mathrm{T}}\right)^{-1} \varphi\left(V_{\mu_{1}}, V_{\mu_{2}}, \ldots, V_{\mu_{M N}}\right)\right),
$$

where $V_{\mu_{j}}:=\sum_{i=1}^{M N}\left[\Pi_{I / O}\right]_{\mu_{i} \mu_{j}} G_{\mu_{i}} G_{\mu_{i}}^{\mathrm{T}} \pi_{I / O}\left(\mu_{i}\right)$.

Corollary 1. For an MSS Markov jump-linear system (5), where $\boldsymbol{\rho}(k)$ is aperiodic and ergodic, if $\boldsymbol{x}_{0}=0$, and $\boldsymbol{w}$ and $\boldsymbol{\rho}$ are independent then

$$
J_{w}=\operatorname{tr}\left(\sum_{j=1}^{M N}\left[\bar{C}_{\mu_{j}} \bar{Q}_{\mu_{j}}\right]\right) .
$$

Proof. For any $k \in \mathbb{Z}^{+}$,

$$
\begin{aligned}
J_{w} & =\lim _{k \rightarrow \infty} E\left\{\boldsymbol{y}^{\mathrm{T}}(k) \boldsymbol{y}(k)\right\} \\
& =\lim _{k \rightarrow \infty} E\left\{\operatorname{tr}\left(\tilde{C}_{\boldsymbol{\rho}(k)}^{\mathrm{T}} \tilde{C}_{\boldsymbol{\rho}(k)} \boldsymbol{x}(k) \boldsymbol{x}^{\mathrm{T}}(k)\right)\right\} \\
& =\lim _{k \rightarrow \infty} \operatorname{tr}\left(\sum_{j=1}^{M N}\left(E\left\{\tilde{C}_{\mu_{j}}^{\mathrm{T}} \tilde{C}_{\mu_{j}} \boldsymbol{x}(k) \boldsymbol{x}^{\mathrm{T}}(k) \mathbf{1}_{\left\{\boldsymbol{\rho}(k)=\mu_{j}\right\}}\right\}\right)\right) \\
& =\operatorname{tr}\left(\sum_{j=1}^{M N}\left(\bar{C}_{\mu_{j}} \cdot \lim _{k \rightarrow \infty} E\left\{\boldsymbol{x}(k) \boldsymbol{x}^{\mathrm{T}}(k) \mathbf{1}_{\left\{\boldsymbol{\rho}(k)=\mu_{j}\right\}}\right\}\right)\right) \\
& =\operatorname{tr}\left(\sum_{j=1}^{M N}\left(\bar{C}_{\mu_{j}} \bar{Q}_{\mu_{j}}\right)\right),
\end{aligned}
$$

which completes the proof. 
This corollary and the following lemma are used to produce the main performance theorem.

Lemma 3. For an MSS Markov jump-linear system (5), given any $P=\left(P_{1}, P_{2}, \ldots, P_{M N}\right) \in \mathbb{H}_{M N}^{n}$ :

(a) if $\boldsymbol{w}=0$, and for any $k \in \mathbb{Z}^{+} \boldsymbol{x}_{0}$ and $\boldsymbol{\rho}(k)$ are independent, then

$$
E\left\{\boldsymbol{x}^{\mathrm{T}}(k+1) P_{\boldsymbol{\rho}(k+1)} \boldsymbol{x}(k+1)\right\}=E\left\{\boldsymbol{x}^{\mathrm{T}}(k) \mathcal{L}_{\boldsymbol{\rho}(k)}(P) \boldsymbol{x}(k)\right\} ;
$$

(b) if $\boldsymbol{x}_{0}=0$, and for any $k \in \mathbb{Z}^{+} \boldsymbol{w}(k)$ and $\boldsymbol{\rho}(k)$ are independent, then

$$
E\left\{\boldsymbol{x}^{\mathrm{T}}(k+1) P_{\boldsymbol{\rho}(k+1)} \boldsymbol{x}(k+1)\right\}=E\left\{\boldsymbol{x}^{\mathrm{T}}(k) \mathcal{L}_{\boldsymbol{\rho}(k)}(P) \boldsymbol{x}(k)\right\}+E\left\{\operatorname{tr}\left(\tilde{G}_{\boldsymbol{\rho}(k)}^{\mathrm{T}} P_{\boldsymbol{\rho}(k+1)} \tilde{G}_{\boldsymbol{\rho}(k)}\right)\right\} .
$$

Proof. See Ref. 31.

The main results concerning output performance are given next. Specifically, it is shown that $J_{0}$ and $J_{w}$ can be concisely written in terms of generalized observability Gramians.

Theorem 4. Consider an MSS Markov jump-linear system (5), where $\boldsymbol{\rho}(k)$ is aperiodic and ergodic.

(a) If $\boldsymbol{w}=0$, and $\boldsymbol{x}_{0}$ and $\boldsymbol{\rho}$ are independent, then the mean output energy is

$$
J_{0}=E\left\{\boldsymbol{x}_{0}^{\mathrm{T}}\left[\sum_{k=0}^{\infty} \mathcal{L}_{\boldsymbol{\rho}_{0}}^{k}(\bar{C})\right] \boldsymbol{x}_{0}\right\}=\operatorname{tr}\left(X_{0} Q_{0}\right),
$$

where $X_{0}:=E\left\{\boldsymbol{x}_{0} \boldsymbol{x}_{0}^{\mathrm{T}}\right\}, Q_{0}:=E\left\{\sum_{k=0}^{\infty} \mathcal{L}_{\boldsymbol{\rho}_{0}}^{k}(\bar{C})\right\}$, and $\mathcal{L}^{k}$ denotes the composition of $\mathcal{L} k$ times with $\mathcal{L}^{0}(\bar{C}):=\bar{C}$.

(b) If $\boldsymbol{x}_{0}, \boldsymbol{w}$ and $\boldsymbol{\rho}$ are independent, then the mean output power is

$$
J_{w}=\lim _{k \rightarrow \infty} E\left\{\operatorname{tr}\left(\sum_{i=0}^{k-1}\left[\tilde{G}_{\boldsymbol{\rho}(k-i-1)}^{\mathrm{T}} \mathcal{L}_{\boldsymbol{\rho}(k-i)}^{i}(\bar{C}) \tilde{G}_{\boldsymbol{\rho}(k-i-1)}\right]\right)\right\}=\operatorname{tr}\left(E\left\{\bar{G}_{\boldsymbol{\rho}_{s}} \tilde{Q}_{\boldsymbol{\rho}_{s}}\right\}\right),
$$

where $\bar{G}_{\boldsymbol{\rho}_{s}}:=\tilde{G}_{\boldsymbol{\rho}_{s}} \tilde{G}_{\boldsymbol{\rho}_{s}}^{\mathrm{T}}$ and $\tilde{Q}_{\boldsymbol{\rho}_{s}}:=\mathcal{E}_{\boldsymbol{\rho}_{s}}\left(\sum_{k=0}^{\infty} \mathcal{L}^{k}(\bar{C})\right)$.

Proof. See Refs. 31,32.

From Lemma 1, the following result concerning output performance of the stochastic hybrid system (4) is easily verified.

Lemma 4. The output performance metrics $J_{0}$ and $J_{w}$ of the stochastic hybrid system (4) are equivalent to that of the model-equivalent Markov jump-linear system (5).

The next sections demonstrate how to apply the stability and performance tools developed in this section to analyze the output tracking performance of an aircraft with a recoverable flight control system.

\section{Modeling an Aircraft System in Closed-Loop with an RCS}

$\mathrm{T}^{\mathrm{N}}$ this section, models for a Boeing 737 flight control system in closed-loop with an RCS are developed. These mathematical models are used in the subsequent section to analyze the tracking performance of the system.

\section{A. System Identification of the Boeing 737 Simulation Model with an RCS}

The first step in the model building process was to identify two state space models for a Boeing 737 in level flight: one for the nominal mode $\Sigma_{n}:\left(A_{n}, G_{n}, C_{n}\right)$ and one for all the recovery modes $\Sigma_{r}:\left(A_{r_{i}}, G_{r_{i}}, C_{r_{i}}\right)$, $i=1,2, \ldots, 6$, simply denoted as $\Sigma_{r}:\left(A_{r}, G_{r}, C_{r}\right)$. I/O data was collected for identification using the nonlinear Boeing 737 Simulink model described in Ref. 33. The control characteristics of the RCS are discussed in Refs. 14,15. The only "inputs" in this case are the noise signals, which are used in the Simulink code to drive the Dryden wind gust model. The output signal was chosen to be the altitude of the aircraft. 
The function pem from MATLAB's System Identification Toolbox was used for identifying the models. Seventeen sets of data containing 2,000 samples each were used for building the nominal model, and another 35 sets of data were used to verify the model. The identified model for the nominal mode $\Sigma_{n}$ was an eighthorder system. Since the recovery mode is usually unstable, it was assumed that the system normally operates in this mode for a short period of time. Therefore, 190 data sets with only 200 samples per set were used for identification. Another 35 sets were used for verification. The identified model of the recovery mode $\Sigma_{r}$ was sixth-order.

\section{B. The Switched System}

It is only possible to switch between $\Sigma_{n}$ and $\Sigma_{r}$ if their respective state space coordinate systems are the same. But here not even their dimensions are equivalent. To remedy the situation, $\Sigma_{r}$ was embedded into an eighthorder system in such a way that the new system, $\hat{\Sigma}_{r}$, had the same controllability indices as $\Sigma_{n}$, specifically, $\{3,3,2\}$. Therefore, each system can be transformed to the same Brunovsky form, ${ }^{34}$ and the switching can be done (formally) by switching between state space gain matrices, $\hat{K}_{n}$ and $\hat{K}_{r}$, and input transformation matrices, $\hat{L}_{n}$ and $\hat{L}_{r}$. Specifically, Fig. 7 shows how the Brunovsky canonical form acts as a "bridge" between the controller canonical forms of the two modes. $\Sigma_{n}$ is transformed into its controller canonical form $\hat{\Sigma}_{n}$ by $T_{n} . \Sigma_{r}$ is transformed into its controller canonical form $\widehat{\Sigma}_{r}:\left(\widehat{A}_{r}, \widehat{G}_{r}, \widehat{C}_{r}\right)$ by $T_{r}$. Then two stable states are added to $\bar{\Sigma}_{r}$ to produce an eighth-order system $\hat{\Sigma}_{r}$. Now $\hat{\Sigma}_{n}$ and $\hat{\Sigma}_{r}$ can be transformed to the same Brunovsky canonical form $\left(A^{\circ}, G^{\circ}\right)$ by $\left(\hat{K}_{n}, \hat{L}_{n}\right)$ and $\left(\hat{K}_{r}, \hat{L}_{r}\right)$, respectively, since $A^{\circ}=\hat{A}_{n}-\hat{G}_{n} \hat{L}_{n} \hat{K}_{n}=\hat{A}_{r}-\hat{G}_{r} \hat{L}_{r} \hat{K}_{r}$ and $G^{\circ}=\hat{G}_{n} \hat{L}_{n}=\hat{G}_{r} \hat{L}_{r}$. The switching between $\hat{\Sigma}_{n}$ and $\hat{\Sigma}_{r}$

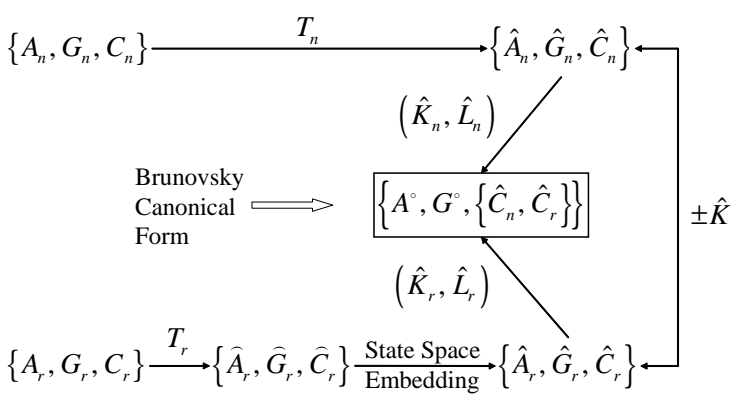

Figure 7. The Brunovsky canonical form acts as a "bridge" between the state space models for the two system modes $\Sigma_{n}$ and $\Sigma_{r}$. can be done using $\hat{K}:=\hat{K}_{n}-\hat{K}_{r}, \hat{L}_{n}$ and $\hat{L}_{r}$, because $\hat{A}_{n}=\hat{A}_{r}+G^{\circ} \hat{K}$ and $\hat{G}_{n}=\hat{G}_{r} \hat{L}_{r} \hat{L}_{n}^{-1}$.

\section{Rollback Recovery Modeling}

The rollback recovery process can be modeled in a variety of ways. The most accurate approach models the actual freezing of the control signals, the rolling back of the control data, the logic of the recovery process, and the delay introduced into the feedback loop. Such an approach is described in Refs. 11,35 for the simplified longitudinal dynamics of the F-16. The complexity of the Boeing 737 nonlinear simulation model in Ref. 33 required that a much simpler approach be taken: only the rollback recovery delay was modeled using either an FSM or an SFSA. Experiments showed that approximately $80 \%$ of the recovery periods of the RCS were six frames in length ( 1 frame $=0.05 \mathrm{sec}$ ) and $20 \%$ were five frames. Any request for a recovery during an active recovery process was ignored. This information was encoded into an FSM by assuming that all the recovery periods were six frames. For better accuracy, an SFSA was also designed to match the recovery length probability distribution. The corresponding FSM $\mathcal{M}=\left(\Sigma_{I}, \Sigma_{S}, \Sigma_{O},\{0,1\}, f, g\right)$ and SFSA $\mathcal{A}=\left(\Sigma_{I}, \Sigma_{S}, \Sigma_{O},[0,1], f, g\right)$ are shown in Figs. 8 and 9 , respectively. The input process to the FSM/SFSA is a homogeneous, two-state, first-order Markov chain. The probability transition matrix of the Markov chain is $\Pi_{I}$. The set of states for the Markov chain is $\Sigma_{I}=\{0,1\}$, where "0" indicates that no upset was detected and "1" indicates that an upset has been detected. The FSM/SFSA symbol sets are $\Sigma_{S}=\left\{N, R_{1}, \ldots, R_{6}\right\}$ and $\Sigma_{O}=\left\{n, r_{1}, \ldots, r_{6}\right\} . f$ is defined by $\Pi^{0}$ and $\Pi^{1}$, and $g$ is a state-to-output isomorphism. The FSM/SFSA produces at its output the random signal $\boldsymbol{\theta}(k)$, which switches between the symbols " $n$ " and " $r_{i}$ ", $i=1,2, \ldots, 6$ representing the "nominal"

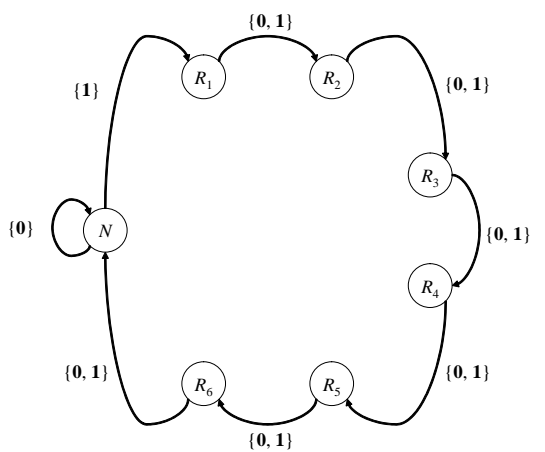

Figure 8. The FSM used to model the rollback recovery process of the RCS. mode and the "recovery" modes, respectively. 


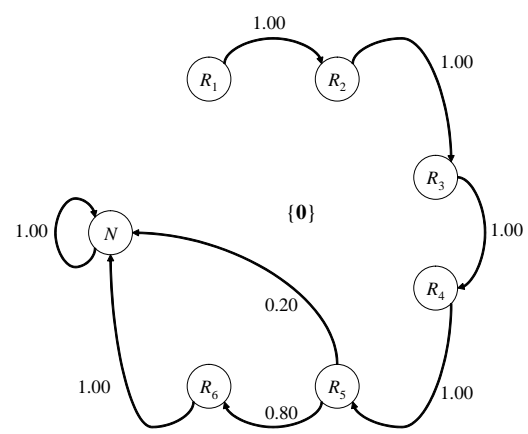

(a) The state transition diagram for input symbol " 0 ", which specifies $\Pi^{0}$.

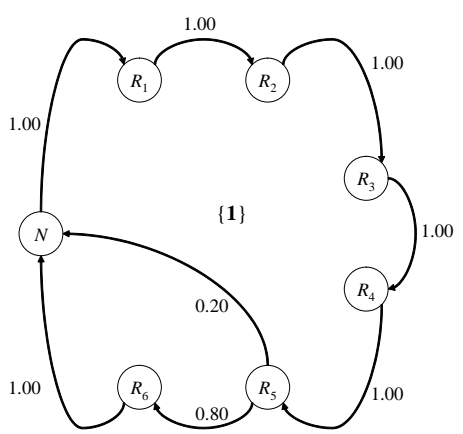

(b) The state transition diagram for input symbol "1", which specifies $\Pi^{1}$.

Figure 9. The SFSA used to model the rollback recovery process of the RCS.

\section{Tracking Performance Analysis of the Boeing 737 Flight System}

SETting the reference input $r(k) \equiv 0$, the nominal (unswitched) system compared against the system with Srecoveries gives rise to an output tracking error described by the following state space model:

$$
\begin{aligned}
\boldsymbol{x}_{e}(k+1) & =A_{e, \boldsymbol{\theta}(k)} \boldsymbol{x}_{e}(k)+G_{e, \boldsymbol{\theta}(k)} \boldsymbol{w}(k), \quad \boldsymbol{x}_{e}(0)=\boldsymbol{x}_{e, 0}, \boldsymbol{\theta}(0)=\boldsymbol{\theta}_{0} \\
\boldsymbol{y}_{e}(k) & =C_{e, \boldsymbol{\theta}(k)} \boldsymbol{x}_{e}(k),
\end{aligned}
$$

where $\boldsymbol{x}_{e}(k)=\left[\begin{array}{ll}\hat{\boldsymbol{x}}^{\mathrm{T}}(k) & \hat{\boldsymbol{x}}_{n}^{\mathrm{T}}(k)\end{array}\right]^{\mathrm{T}}, A_{e, \boldsymbol{\theta}(k)}=\operatorname{diag}\left(\hat{A}_{\boldsymbol{\theta}(k)}, \hat{A}_{n}\right), G_{e, \boldsymbol{\theta}(k)}=\left[\begin{array}{ll}\hat{G}_{\boldsymbol{\theta}(k)}^{\mathrm{T}} & \hat{G}_{n}^{\mathrm{T}}\end{array}\right]^{\mathrm{T}}$ and $C_{e, \boldsymbol{\theta}(k)}=$ $\left[\begin{array}{ll}\hat{C}_{\boldsymbol{\theta}(k)} & -\hat{C}_{n}\end{array}\right] . \hat{\boldsymbol{x}}(k)$ and $\hat{\boldsymbol{x}}_{n}(k)$ are the state vectors of the switched and nominal closed-loop systems; $\boldsymbol{w}(k)$ is a zero-mean white noise process used to generate a wind input; and $\boldsymbol{y}_{e}(k)$ is the closed-loop output tracking error. The models $\hat{\Sigma}_{n}$ and $\hat{\Sigma}_{r}$ described in Subsection IV-B were used to build the 16th-order system (8). The Markov cross chain process $\boldsymbol{\rho}(k)$ has transition probability matrix

$$
\Pi_{I / O}=\operatorname{diag}\left(\Pi^{0}, \Pi^{1}\right) \cdot\left(\Pi_{I} \otimes I_{7}\right) .
$$

Setting $\Pi_{I}=\left(\begin{array}{cc}1-\left[\Pi_{I}\right]_{01} & {\left[\Pi_{I}\right]_{01}} \\ 1 & 0\end{array}\right)$, where parameter $\left[\Pi_{I}\right]_{01}$ is the probability of an upset, Fig. 10 shows $r_{\sigma}\left(\mathscr{A}_{2}\right)$

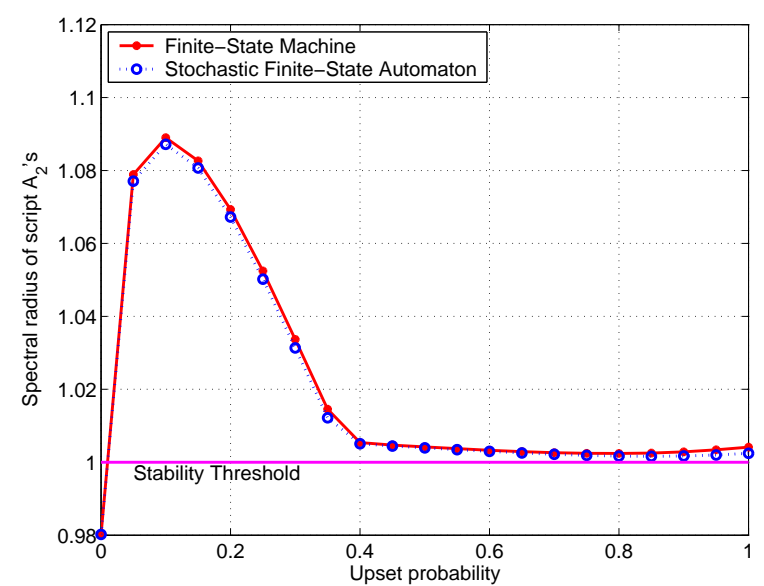

(a) $\left[\Pi_{I}\right]_{01} \in[0,1]$.

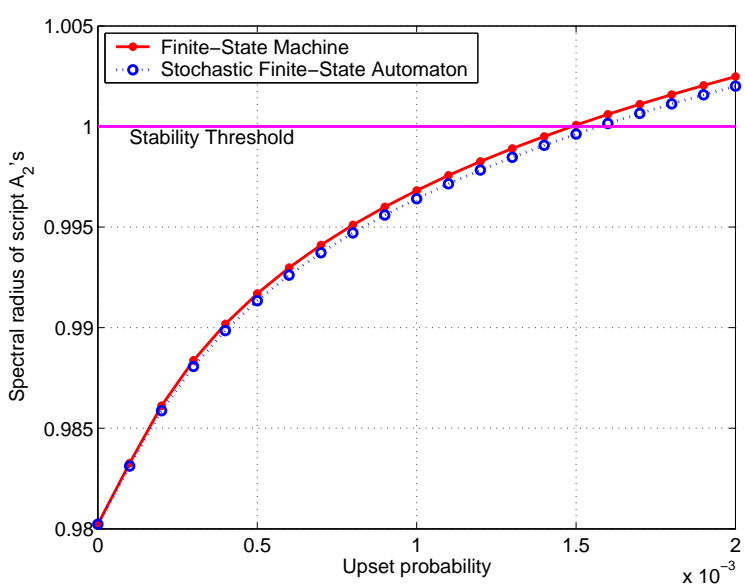

(b) $\left[\Pi_{I}\right]_{01} \in[0,0.002]$.

Figure 10. $r_{\sigma}\left(\mathscr{A}_{2}\right)$ as a function of upset probability for the SFSA stochastic hybrid model and the FSM hybrid dynamical model.

plotted as a function of $\left[\Pi_{I}\right]_{01}$. The SFSA stochastic hybrid model is MSS when $\left[\Pi_{I}\right]_{01}<0.0016$, which is a 
slightly higher stability boundary than that predicted by the FSM hybrid model. When the system is MSS, the predicted mean output tracking error power, $J_{w}$, is shown in Fig. 11 for each model. As it might be expected, the higher the upset probability, the larger the output tracking error power. In addition, the error power is unbounded at the stability boundary.

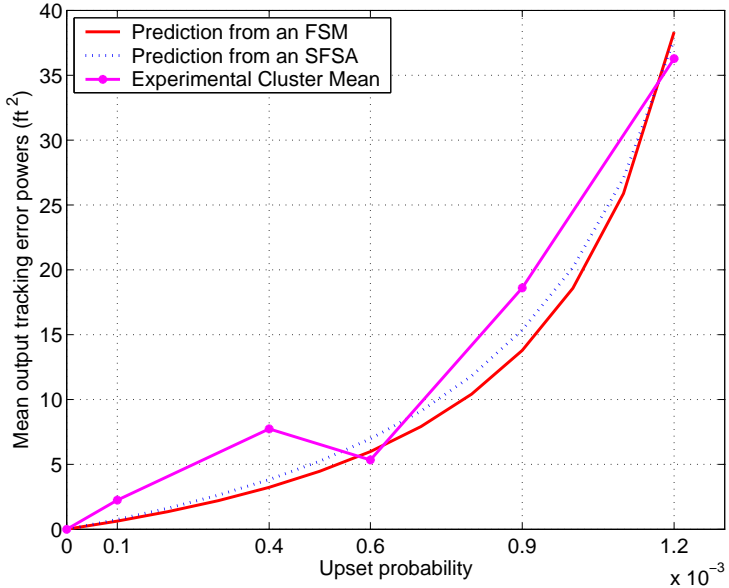

Figure 11. The SFSA/FSM-predicted mean output tracking error powers as a function of upset probability and the experimental results for six specific upset probabilities.

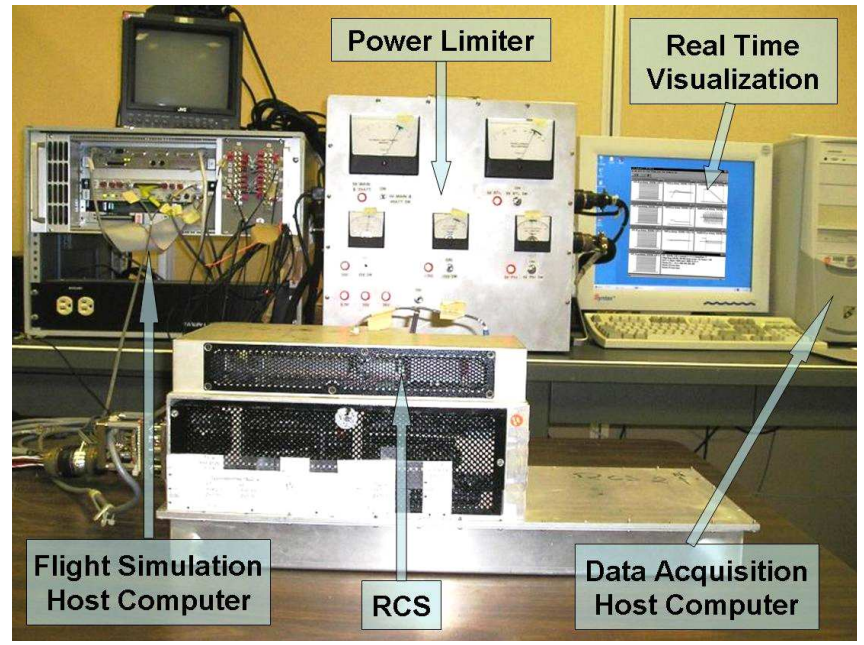

Figure 12. The RCS experimental environment in the SAFETI Laboratory at NASA Langley.

\section{Model Validation Using the NASA Experimental Data}

series of experiments was conducted in a simulated neutron environment at the NASA Langley Research Center's SAFETI Laboratory. Fig. 12 shows the experimental testbed and the RCS developed by Honeywell. The RCS flight control system was connected in closed-loop with a Boeing 737 flight simulation system running on a separate host computer. The input reference signals were set to maintain straight and level flight at a cruising altitude of 34,000 feet. A data acquisition system was maintained on a third computer system. It collected flight data during the simulation. Neutron interactions were simulated by triggering rollback recoveries according to a pre-determined Markov upset process while the aircraft flew in $1 \mathrm{ft} / \mathrm{sec}$ (light) winds. High wind conditions were not tested since they can excite nonlinear modes in the aircraft dynamics, which are not modeled in the present jump-linear framework. Data was collected for 60 one-hour (real time) flights.

Ten sets of experimental data were collected for each of the following six upset probabilities $\left[\Pi_{I}\right]_{01}: 0,0.0001$, $0.0004,0.0006,0.0009$, and 0.0012. In each case, different sample functions from the corresponding Markov process $\boldsymbol{\nu}(k)$ were used to supply the RCS with a series of recovery requests for the closed-loop system. The ten experimental output tracking error powers for each value of $\left[\Pi_{I}\right]_{01}$ are shown in Fig. 13. Cluster analysis, a tool for analyzing under sampled data sets, was used to exclude the outliers for each probability. ${ }^{36}$ Specifically, as shown in Fig. 14, hierarchical clustering diagrams were used based on the Euclidean distances between the measured output powers. The dashed lines denote experimental data which was discarded because it was far away from the main clusters (indicated in solid lines). Here the one or two highest links were removed if they were approximately four times higher than the other links. The average output tracking error powers for the six proba-

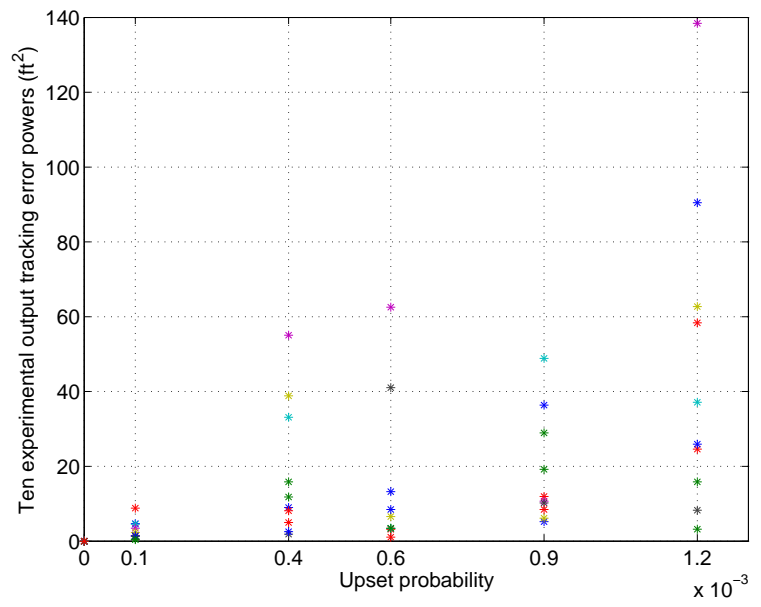

Figure 13. The distribution of ten output tracking error powers for six specific upset probabilities. 
bilities were computed empirically from the experimental output data within the main cluster. The results are shown in Fig. 11 and compared against the theoretical predictions using both the SFSA and the FSM. The SFSA prediction is superior, as it is slightly closer to the experimental curve.

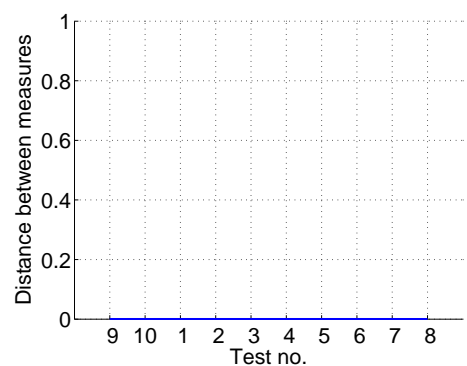

(a) $\left[\Pi_{I}\right]_{01}=0$.

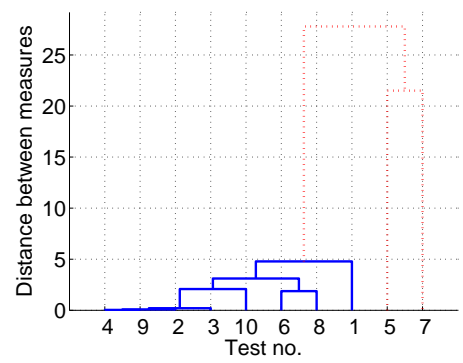

(d) $\left[\Pi_{I}\right]_{01}=0.0006$.

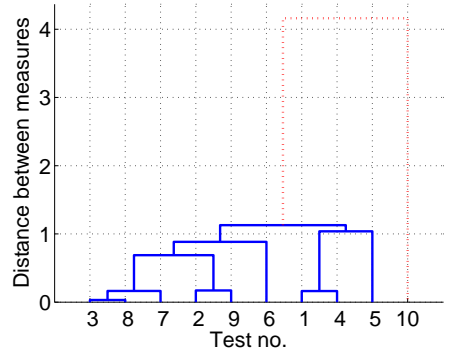

(b) $\left[\Pi_{I}\right]_{01}=0.0001$.

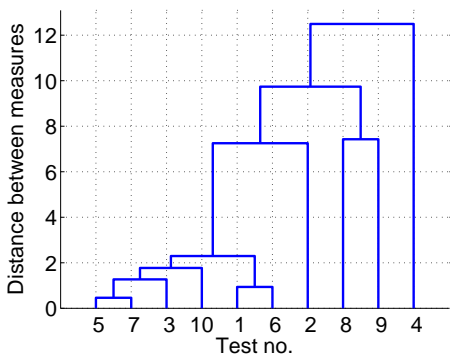

(e) $\left[\Pi_{I}\right]_{01}=0.0009$.

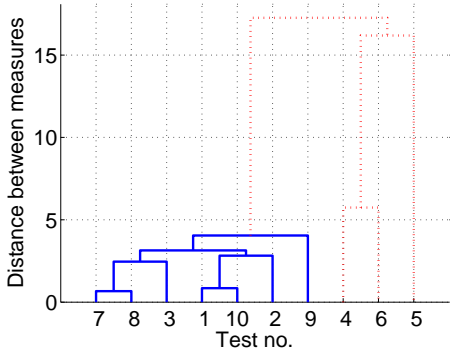

(c) $\left[\Pi_{I}\right]_{01}=0.0004$.

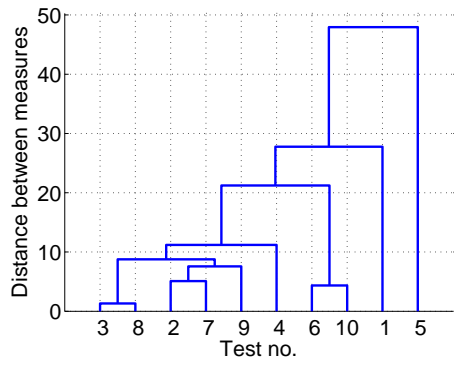

(f) $\left[\Pi_{I}\right]_{01}=0.0012$.

Figure 14. The cluster analysis diagrams for six specific upset probabilities.

\section{Conclusions}

$\mathrm{T}_{\mathrm{N}}^{\mathrm{N}}$ this paper, a class of stochastic hybrid models was introduced for modeling recoverable closed-loop 1systems subject to Markovian upset processes. A mean-square stability criteria was developed using a model-equivalent Markov jump-linear system. Output performance was characterized in terms of a new generalized observability Gramian. The model was then applied to a Boeing 737 in closed-loop with an RCS subject to neutron induced SEU's. Performance predictions were validated using data from a simulated neutron environment at NASA Langley. In the future, these analysis tools will be used to rescale performance estimates derived from real (accelerated) neutron tests conducted at Los Alamos. This will allow the FAA to assess any potential safety hazards due to SEU's for aircraft flying under normal atmospheric conditions.

\section{Acknowledgments}

This research was supported by the NASA Langley Research Center under contracts NCC-1-03026 and NNL04AA03A, and by the National Science Foundation under grant CCR-0209094. The authors also wish to acknowledge the generous assistance of Mr. Edward F. Hogge, Computer Systems Analyst, Lockheed Martin Engineering and Sciences, for conducting the RCS experiments at NASA and providing the data for this paper.

\section{References}

${ }^{1}$ Mendell, R. B. and Korff, S. A., "Fast-Neutron Flux in the Atmosphere," J. Geophys. Res., Vol. 68, No. 19, 1963, pp. $5487-5495$.

${ }^{2}$ Ziegler, J. F., "Terrestrial Cosmic Rays," IBM J. Res. Develop., Vol. 40, No. 1, 1996, pp. 19-39.

${ }^{3}$ Normand, E. and Baker, T. J., "Altitude and Latitude Variations in Avionics SEU and Atmospheric Neutron Flux," IEEE Trans. Nucl. Sci., Vol. 40, No. 6, 1993, pp. 1484-1490. 
${ }^{4}$ Messenger, G. C. and Ash, M. S., The Effects of Radiation on Electronic Systems, Van Nostrand Reinhold, New York, 2nd ed., 1992.

${ }^{5}$ Tribble, A. C., The Space Environment: Implications for Spacecraft Design, Princeton University Press, Princeton, NJ, 1995.

${ }^{6}$ Messenger, G. C. and Ash, M. S., Single Event Phenomena, Chapman \& Hall, New York, 1997.

${ }^{7}$ Normand, E., "Single-Event Effects in Avionics," IEEE Trans. Nucl. Sci., Vol. 43, No. 2, 1996, pp. $461-474$.

${ }^{8}$ O'Gorman, T. J., Ross, J. M., Taber, A. H., Ziegler, J. F., Muhlfeld, H. P., Montrose, C. J., Curtis, H. W., and Walsh, J. L., "Field Testing for Cosmic Ray Soft Errors in Semiconductor Memories," IBM J. Res. Develop., Vol. 40, No. 1, 1996, pp. $41-50$.

${ }^{9}$ Ziegler, J. F., Muhlfeld, H. P., Montrose, C. J., Curtis, H. W., O'Gorman, T. J., and Ross, J. M., "Accelerated Testing for Cosmic Soft-Error Rate," IBM J. Res. Develop., Vol. 40, No. 1, 1996, pp. 51-72.

${ }^{10}$ Gray, W. S., González, O. R., and Doğan, M., "Stability Analysis of Digital Linear Flight Controllers Subject to Electromagnetic Disturbances," IEEE Trans. Aerosp. Electron. Syst., Vol. 36, No. 4, 2000, pp. 1204-1218.

${ }^{11}$ González, O. R., Gray, W. S., and Tejada, A., "Analytical Tools for the Design and Verification of Safety Critical Control Systems," 2001 SAE Transact.-J. Aerosp., Vol. 110, No. 1, 2002, pp. 481-490.

${ }^{12}$ Hess, R., "Computing Platform Architectures for Robust Operation in the Presence of Lightning and Other Electromagnetic Threats," 16th Digital Avionics Systems Conf. Proceedings, Irvine, California, 1997, pp. 4.3-9-16.

${ }^{13}$ Hess, R., "Options for Aircraft Function Preservation in the Presence of Lightning," Proc. 1999 International Conf. Lightning Static Electricity, Toulouse, France, 1999.

${ }^{14}$ Malekpour, M. and Torres, W., "Characterization of a Recoverable Flight Control Computer System," Proc. 1999 IEEE Int. Conf. Contr. Appl., Kohala Coast-Island of Hawaii, Hawaii, 1999, pp. 1519-1524.

${ }^{15}$ Malekpour, M. and Torres, W., "Characterization of a Flight Control Computer with Rollback Recovery," 19th Digital Avionics Systems Conf. Proceedings, Philadelphia, Pennsylvania, 2000, pp. 3.C.4-1-8.

${ }^{16}$ Narasimhan, R., Rosenkrantz, D. J., and Ravi, S. S., "Early Comparison and Decision Strategies for Datapaths that Recover from Transient Faults," IEEE Trans. Circuits Syst.-I: Fundam. Theory Appl., Vol. 44, No. 5, 1997, pp. 435-438.

${ }^{17}$ Ranganathan, A. and Upadhyaya, S. J., "Performance Evaluation of Rollback-Recovery Techniques in Computer Programs," IEEE Trans. Reliab., Vol. 42, No. 2, 1993, pp. 220-226.

${ }^{18}$ Costa, O. L. V. and Marques, R. P., "Mixed $H_{2} / H_{\infty}$-Control of Discrete-Time Markovian Jump Linear Systems," IEEE Trans. Automat. Contr., Vol. 43, No. 1, 1998, pp. 95-100.

${ }^{19}$ Costa, O. L. V. and Fragoso, M. D., "Stability Results for Discrete-Time Linear Systems with Markovian Jumping Parameters," J. Math. Anal. Appl., Vol. 179, 1993, pp. 154-178.

${ }^{20}$ do Val, J. B. R. and Costa, E. F., "Numerical Solution for Linear-Quadratic Control Problems of Markov Jump Linear Systems and Weak Detectability Concept," J. Optim. Theory Appl., Vol. 114, No. 1, 2002, pp. 69-96.

${ }^{21}$ Ünsal, C., Intelligent Navigation of Autonomous Vehicles in an Automated Highway System: Learning Methods and Interacting Vehicles Approach, Ph.D. thesis, The Bradley Department of Electrical and Computer Engineering, Virginia Polytechnic Institute and State University, 1997.

${ }^{22}$ Tou, J. T., "Stochastic Automata and Discrete Systems Theory," Applied Automata Theory, edited by J. T. Tou, Electrical Science Series, Academic Press, New York, 1968, pp. 55-80.

${ }^{23}$ Lunze, J. and Nixdorf, B., "Representation of Hybrid Systems by Means of Stochastic Automata," Math. Comput. Modelling Dyn. Syst., Vol. 7, No. 4, 2001, pp. 383-422.

${ }^{24}$ Patilkulkarni, S., Herencia-Zapana, H., Gray, W. S., and González, O. R., "On the Stability of Jump-Linear Systems Driven by Finite-State Machines with Markovian Inputs," Proc. 2004 Amer. Control Conf., Boston, Massachusetts, 2004, pp. $2534-2539$.

${ }^{25}$ Patilkulkarni, S., Stability Analysis of Jump-Linear Systems Driven by Finite-State Machines with Markovian Inputs, Ph.D. thesis, Department of Electrical and Computer Engineering, Old Dominion University, 2004.

${ }^{26}$ Horbacz, K., "Random Dynamical Systems with Jumps," J. Appl. Prob., Vol. 41, No. 3, 2004, pp. 890-910.

${ }^{27}$ Arnold, L. and Kliemann, W., "Qualitative Theory of Stochastic Systems," Probability Analysis and Related Topics, edited by A. T. Bharucha-Reid, Vol. 3, Academic Press, Inc., New York, 1983, pp. 1-79.

${ }^{28}$ Zhang, H., Gray, W. S., and González, O. R., "Performance Analysis of Digital Flight Control Systems with Rollback Error Recovery Subject to Simulated Neutron-Induced Upsets," IEEE Trans. Control Syst. Technol., 2005, under review.

${ }^{29}$ Costa, O. L. V. and Marques, R. P., "Robust $H_{2}$-Control for Discrete-Time Markovian Jump Linear Systems," Int. J. Control, Vol. 73, No. 1, 2000, pp. 11-21.

${ }^{30}$ Ling, Q. and Lemmon, M. D., "Soft Real-Time Scheduling of Networked Control Systems with Dropouts Governed by a Markov Chain," Proc. 2003 Amer. Control Conf., Denver, Colorado, 2003, pp. 4845-4850.

${ }^{31}$ Gray, W. S., Zhang, H., and González, O. R., "Closed-Loop Performance Measures for Flight Controllers Subject to Neutron-Induced Upsets," Proc. 42nd IEEE Conf. Decision Control, Maui, Hawaii, 2003, pp. 2465-2470.

${ }^{32}$ Zhang, H., Gray, W. S., and González, O. R., "Performance Analysis of Recoverable Flight Control Systems Using Hybrid Dynamical Models," Proc. 2005 Amer. Control Conf., Portland, Oregon, 2005, pp. 2787-2792.

${ }^{33}$ Hogge, E. F., "B-737 Linear Autoland Simulink Model," Tech. Rep. CR-2004-213021, NASA, Langley Research Center, Hampton, Virginia, 2004.

${ }^{34}$ Kailath, T., Linear Systems, Prentice-Hall Information and System Sciences Series, Prentice-Hall, Inc., Englewood Cliffs, New Jersey, 1980.

${ }^{35}$ Tejada, A., Analysis of Error Recovery Effects on Digital Flight Control Systems, Master's thesis, Department of Electrical and Computer Engineering, Old Dominion University, 2002.

${ }^{36}$ Hawkins, D. M., Identification of Outliers, Vol. 13 of Monographs on Applied Probability and Statistics, Chapman \& Hall, London, 1980. 